\title{
AUTOTRANSFUSÃO DE PRÉ-COLETA IMEDIATA
}

\section{BLOOD AUTO-TRANSFUSION OF PREVIOUS PRÉ-COLLECTION OF BLOOD}

\author{
Levão Bogossian, ECBC-RJ'1; Aníbal da Torre Bogossian²
}

\section{AUTOTRANSFUSÃO DE PRÉ-COLETA IMEDIATA}

A autotransfusão é um método terapêutico que consiste na reintrodução do sangue do paciente em suas próprias veias, ou seja, é uma transfusão autóloga. Ela difere das transfusões sangüíneas comuns realizadas com o sangue de outros seres humanos: transfusões homólogas.

A autotransfusão é prática muito valorizada atualmente por seus benefícios econômicos e, principalmente, por sua maior segurança clínica. Os graves riscos das transfusões homólogas reforçam o valor e a importância da autotransfusão.

Vários são os tipos de autotransfusão atualmente existentes que, ao nosso ver, devem ser agrupados em duas modalidades básicas ${ }^{1-5}$.

1. Autotransfusões de emergência - Reinfusão.

2. Autotransfusões de pré-depósito - Programadas.

Nesta revisão abordaremos as vantagens e indicações das autotransfusões pré-depósito que podem ser de duas modalidades, de acordo com a fase em que a pré-coleta (ou coletas) é realizada.

\section{Autotransfusões Imediatas - Reinfusão}

Nesse grupo de autotransfusões, o sangue derramado em uma hemorragia qualquer do paciente seja no período pré, per ou pós-operatório, é coletado por meios variados e imediatamente reintroduzido em sua veia. Trata-se de uma autotransfusão de emergência porque, na maioria das vezes, o paciente está agudamente desfalcado daquele volume sanguiíneo perdido e precisa ser reposto de imediato. Essa prática é habitualmente designada, em nosso meio, por reinfusão ${ }^{1-4,6,7}$.

Elas são basicamente indicadas em atos cirúrgicos de pacientes que sofreram hemorragias prévias ou na própria operação que está sendo realizada.

As principais modalidades de reinfusão atualmente existentes são:

a) Reinfusão transoperatória de sangue acumulado em cavidades serosas por hemorragias anteriores (hemoperitônio e hemotórax ) ; b) Reinfusão transoperatória de sangue derramado durante os procedimentos cirúrgicos muito sangrantes ${ }^{1,8-10}$; c) Reinfusão pós-operatória (utiliza sangue que escoa por drenos nos pós-operatórios) ${ }^{1,7,11,12}$; d) Reinfusão do sangue contido nos órgãos extirpados durante o ato operatório ${ }^{7}$. Este método de autotransfusão foi por nos proposto em 1989. Ele utiliza o sangue contido no órgão retirado, durante a operação, para ser reinfundido de imediato no próprio paciente. Este método é utilizado principalmente em esplenectomias ${ }^{1,13-15}$. Lógica e evidentemente, não deve ser usado em órgãos infectados ou portadores de neoplasia maligna.

2.Autotransfusões de Pré-Depósito - Programadas

A autotransfusão de pré-depósito consiste na précoleta prévia do sangue do paciente para reinfundi-lo no momento em que dele necessitar.

Elas são programadas em duas modalidades: a) Précoleta múltipla iniciada de seis a 28 dias antes da data da operação e b) Pré-coleta imediata realizada de 10 a 30 minutos antes do início do ato operatório (Este é o tema básico da presente revisão).

A absoluta segurança da autotransfusão de pré-depósito e suas inúmeras vantagens sobre as transfusões homólogas têm sido constantemente alardeadas na literatura mundial, com a adoção do método em várias áreas cirúrgicas, principalmente na ortopédica e na cardíaca, sendo, também, muito usada em cirurgia vascular, abdominal, plástica (lipectomias e lipoaspiração), ginecológica, obstétrica, neurocirurgia, e também nas operações para transplantes de órgãos ${ }^{1,14,16-26}$.

A fantástica disseminação atual de programas de pré-depósito trouxe também o grande benefício de reduzir enormemente as necessidades das transfusões homólogas ${ }^{24,25,27}$ e está propiciando aumento dos estoques de sangue nos bancos de sangue. Estão também surgindo propostas de aproveitamento do sangue pré-doado e não utilizado, no paciente doador, por desnecessidade, tornando-o um sangue homólogo para transfusões em outros enfermos ${ }^{28}$ ou como fonte de processamento de diferentes derivados.

Inúmeras são as vantagens da autotransfusão de pré-depósito. Elas serão enumeradas e se aplicam às duas modalidades básicas. Em seguida, cada modalidade será analisada com ênfase especial ao tema básico - Autotransfusão de pré-coleta imediata.

1. Professor Titular Emérito da Faculdade de Medicina da Universidade Federal do Rio de Janeiro - UFRJ.

2. Mestre e Doutor pela Faculdade de Medicina da Universidade Federal do Rio de Janeiro - UFRJ.

Recebido em 02/01/2008

Aceito para publicação em 01/02/2008

Conflito de interesses: nenhum

Fonte de financiamento: nenhuma 


\section{Vantagens das autotransfusões de pré-depósito}

- Ausência dos riscos e das complicações das transfusões homólogas.

- Não estão restritas à disponibilidade de tipo sangüíneo.

- Não são onerosas. O aspecto econômico das autotransfusões deve ser sempre ressaltado, pois seus gastos são sempre muito menores do que os de qualquer transfusão de sangue ou seus derivados.

- Dispensam classificações repetidas e pesquisas de anticorpos de moléstias variadas, que consomem tempo e dinheiro.

- Podem ser praticadas em hospitais ou cidades desprovidas de maiores recursos, inclusive naquelas que não dispõem de banco de sangue. Basta uma geladeira e bolsas de coleta de sangue (hemobags), que já trazem consigo os preservativos, para se fazer um bom pré-depósito.

- São desprovidas de antigenicidade ${ }^{29}$; não provocam sangramentos por coagulopatias induzidas; não produzem bloqueio alveolocapilar, acidose ou necrose tubular.

- Conservam o conteúdo de 2-3 DPG de suas hemácias.

- Não provocam depressão imunológica, nem aloimunização.

- Podem ser aplicadas em pacientes adeptos de religiões que proíbem transfusões sangüíneas regulares, como os "Testemunhas de Jeová".

- Reduzem enormemente as necessidades de sangue homólogo em diferentes especialidades $1,5,6,7,14,18,19,30-34$.

- Podem constituir fonte de transfusão homóloga para outros pacientes quando o pré-depositado não for utilizado na durante o ato operatário. Esse dado é de suma importância econômica porque, além de constituir fonte de transfusão homóloga e reduzir as necessidades desta, as pré-coletas não usadas podem ser fracionadas e aproveitadas sob diversas formas de derivados e, até, na fabricação de cola de fibrina.

- A simplicidade e facilidade da aplicação do prédepósito (pré-coleta) se igualam ao único item vantajoso das transfusões homólogas.

\section{A- Autotransfusão de pré-depósito por pré-coleta} múltipla

Embora este não seja, também, o tema básico desta revisão, faremos um comentário sobre as qualidades da précoleta múltipla para a valorização da pré-coleta imediata.

Na pré-coleta múltipla, o número de doações e o volume a ser coletado são variáveis, de acordo com o tipo de intervenção cirúrgica a ser praticada e com o estado prévio do paciente. Os intervalos entre doações variam de quatro a sete dias, para permitir a recuperação hemática do paciente. As doações múltiplas são as mais freqüentemente utilizadas e variam de duas a quatro (ou mais) unidades de $450 \mathrm{ml}$, iniciando-se com duas a quatro semanas de antecedência da da operação.

A anemia e a hipovolemia decorrentes das pré-doações são corrigidas da seguinte maneira: a hipovolemia é facilmente restaurada pela infusão imediata de soluções salinas ou coloidais, em igual volume ao retirado. Quando se usa ex- clusivamente soluções salinas, os volumes de reposição devem ser maiores, porque elas se eliminam mais rapidamente. Já as soluções coloidais, como a gelatina de osseína (Haemacelâ) ou hidroxietil amido, que têm maior permanência, podem ser usadas em iguais volumes.

A anemia é compensada nos intervalos das doações com o uso de doses elevadas (200mg/dia) de ferro injetável ou oral, complementadas com vitaminas e possivelmente com ativadores da eritropoiese como a eritripoietina recombinante humana (rHuEPO). Os resultados oferecidos por essa substância estimuladora são de primeira qualidade ${ }^{1,28}$. O único senão para o nosso meio, reside no alto preço do produto. Países do Segundo Mundo têm de se conformar com a simples reposição de ferro, vitaminas e ácido fólico. Junqueira ${ }^{28}$ nos mostrou que é possível realizar programas muito bons de pré-depósito apenas com essa reposição elementar (ferro e vitaminas).

A grande problemática que envolvia os programas de pré-depósito múltiplo era a do envelhecimento das unidades doadoras no início das coletas. Porém, com o surgimento da proposta de Ascari ${ }^{35}$ do chamado "salto do sapo", que fazia retornar ao paciente as unidades velhas colhidas, para realizar novas coletas de sangue mais fresco e evitar espoliações maiores, este problema foi bem contornado (Figura1).

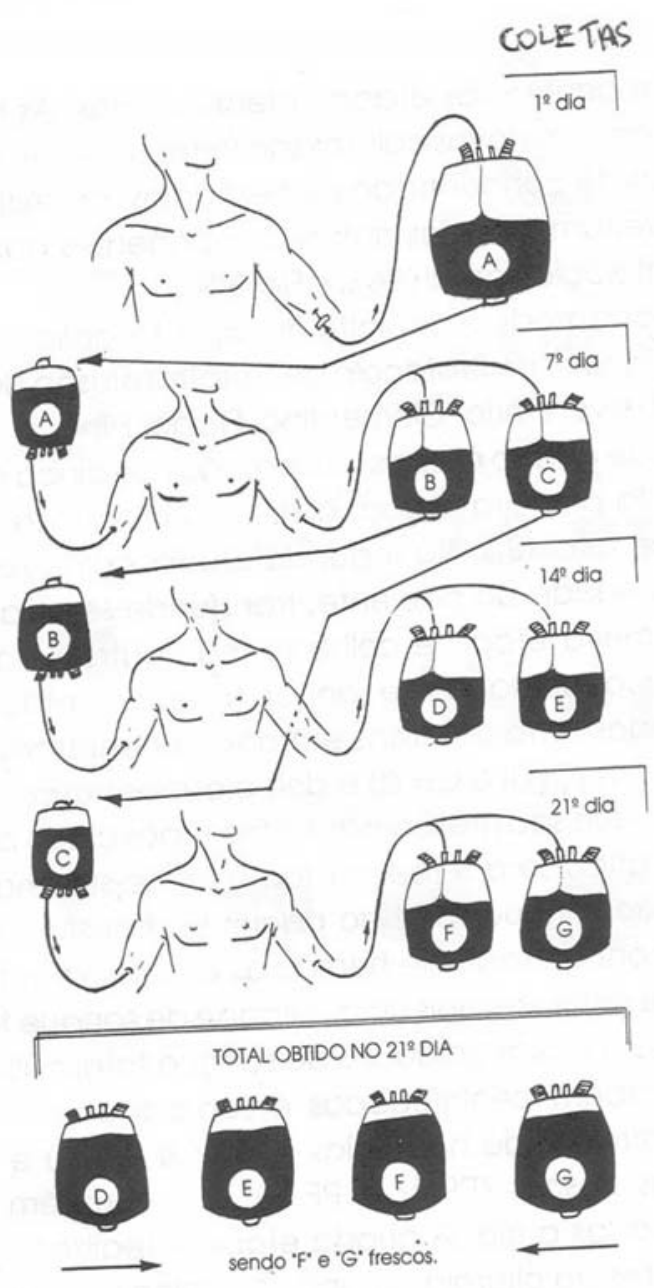

Figura 1 - Esquema do "salto do sapo". 


\section{B - Autotransfusão de pré-coleta imediata (pré-de- pósito para uso imediato)}

Este tipo de autotransfusão era denominado "hemodiluição isovolêmica pré-operatória imediata" porque durante, ou mesmo antes, a pré-coleta era acompanhada de uma reposição volêmica volumosa feita principalmente com soluções salinas para recompor o volume do sangue coletado. Nesta prática o sangue assim pré-coletado continha menos hemácias.

Com base neste problema, em 1996, um ano após a publicação do nosso livro, Manual de Autotransfusão, passamos a evitar a "hemodiluição isovolêmica" fazendo a "précoleta imediata" sem nenhuma reposição volêmica paralela. A reposição volêmica só é realizada após o encerramento da pré - coleta imediata.

O pré-depósito para uso imediato (tema básico do presente trabalho) consiste na pré-coleta de sangue da veia do paciente, poucos minutos antes da operação, para depois transfundi-lo quando a operação terminar, ou durante a mesma se ocorrer sangramento importante. O sangue é coletado no equipamento clássico de coleta e de administração denominado "Hemobag", que é mantido junto ao paciente (de uma boa veia se pode retirar cerca de $500 \mathrm{ml} \mathrm{em}$ 10 minutos).

A pré-coleta é preferencialmente realizada na sala de operações (S.O.) antes da anestesia, mas pode ser feita 30 minutos antes (no quarto) e conduzida junto com o paciente, quando ele for encaminhado ao centro cirúrgico. Somente ao término da pré-coleta, o paciente é reposto em igual volume, com solução salina ou coloidal, para manutenção da volemia. $\mathrm{Na}$ maioria das vezes, a pré-coleta não prejudica a volemia porque o volume extraído no "hemobag" não ultrapassa a $500 \mathrm{ml}^{1}$. entretanto, alguns autores ${ }^{14,15,18,34}$ estão preferindo pré-coletas maiores para determinados casos, tornando necessária a reposição salina paralela ${ }^{15}$.

A autotransfusão de pré-depósito imediata constitui uma estratégia muito inteligente e bem calçada em bases fisiopatológicas sólidas, que pode ser largamente aplicada. Aliás, a tendência mundial é, inclusive, a de dar preferência a esse tipo de pré-depósito, por ser ele o mais econômico (dispensa conserva) e porque sua única restrição, a coleta de uma só unidade, foi removida, pois já se aumentou o número de unidades colhidas em determinados casos ${ }^{14,15,18,34,36}$. Já está comprovado ${ }^{15}$ que os pacientes toleram bem essas retiradas até o hematócrito de $20 \%$. Caso o hematócrito baixe deste mínimo tolerável, o paciente pode ser imediatamente reposto com seu próprio sangue e com colóides ou salinos que estiverem sendo usados. Na operação cardíaca os cirurgiões estão retirando o sangue do circuito do "by pass" antes do seu acionamento para retransfundi-lo ao término da circulação extracorpórea ${ }^{34}$.

\section{Bases fisiopatológicas da pré-coleta imediata ${ }^{1-5}$}

A pré-coleta imediata está reduzindo o sangramento das incisões do campo operatório. Isto, seguramente, se deve à vasoconstrição microcirculatória.

A pré-coleta não prejudica a estabilidade hemodinâmica porque o volume sangüíneo é mantido pela reposição salina e/ou coloidal que a sucede ${ }^{19}$. O paciente mantém-se, portanto, com alguns parâmetros hemodinâmicos estáveis e outros até aumentados, como a velocidade de fluxo, o retorno venoso, o enchimento diastólico e o débito cardíaco.

Uma pequena redução do hematócrito é observada na pré-coleta imediata $(500 \mathrm{ml})$, mas ela não interfere substancialmente no aporte de oxigênio à célula, uma vez que esta aumenta muito o seu poder de extração de $\mathrm{O}_{2}$ diante de baixos níveis de hemoglobina. Além disso, a curva de dissociação da hemoglobina pende muito para a direita na anemia, fazendo com que o oxigênio seja mais facilmente liberado pelas hemácias para utilização celular.

Estando baixo o hematócrito do paciente durante a operação, todo sangue que ele compulsoriamente perde durante o ato operatório é pobre em hemácias, o que reduz muito os efeitos nocivos da hemorragia cirúrgica. Ao término da operação ele recebe, então, seu sangue coletado, que é bastante rico em hemácias por ter sido coletado antes da hemodiluição.

A diminuição da viscosidade sangüínea produzida pela leve hemodiluição decorrente da pré-coleta facilita a perfusão de órgãos vitais e esta melhora reológica reduz bem a resistência periférica durante o ato operatário.

$\mathrm{O}$ paciente é reinfundido com um sangue fresco e ainda quente, que mantém todos os seus componentes estáveis porque não precisou de meios de conserva. O sangue pré-coletado permanece na sala de operações, sem preservação especial, até seis horas, sem problemas.

Seu custo é bem menor do que o do pré-depósito tradicional de várias coletas, que exige vários comparecimentos do doador e procedimentos de depósito e conserva, de certa forma onerosos.

O risco de trocas ou problemas de erro de etiquetagem diminui muito no pré-depósito imediato porque o sangue coletado não sai da sala de operações.

É também importante relembrar que: a) cada unidade $(450 \mathrm{ml})$ retirada diminui o hematócrito somente em três a $5 \%$; b) os pacientes toleram bem as retiradas, podendo até chegar ao hematócrito de $20 \%$; c) hematócritos de 20 a $30 \%$ das pré-coletas maiores sofrem redução de fatores de coagulação na ordem de $30 \%$ e esta redução não causa problemas hemorrágicos, pois a coagulação sangüínea ainda está preservada quando o paciente tem apenas $15 \%$ de seus fatores ${ }^{15}$.

O temor que aflige aos menos afeitos à pré-coleta imediata, com relação ao choque, hipocoagulabilidade, anemia aguda ou má irrigação coronariana súbita, não procede; desde que se se respeitem as contra-indicações formais, quais sejam: anemia prévia (hematócrito inicial abaixo de 20\%); casos de operação de urgência ocasionadas por perdas sanguineas elevadas; doenças coronarianas graves (que não suportem baixos níveis de hematócritos); presença de infecção e insuficiência renal. No item insuficiência renal, o problema reside no fato de que, se necessária a infusão de grandes volumes de líquidos, eles serão mais lentamente eliminados, podendo provocar hipervolemia quando o sangue previamente retirado for reinfundido. 


\section{CONSIDERAÇÕES FINAIS}

Nossa insistência na prática de autotransfusão data de 1952, desde que propusemos a utilização do método por nós criado ${ }^{2}$, que simplificava a coleta de sangue e a tornava mais dinâmica. Naquela oportunidade, era mínima a divulgação nacional ou internacional da autotransfusão, mas nós constantemente alardeávamos seu valor. Nossa obstinação era freqüentemente contestada sob a alegação de que as modernas transfusões homólogas se haviam tornado muito simples e bastante práticas. Fomos até taxados de retrógrados por insistirmos num método que se fizera penoso diante das facilidades das transfusões. Quando os casos de hepatite (B, C, e não A, não B) e da AIDS transmitidas pela transfusão de sangue homólogo ficaram patentes; todos voltaram a considerar, então, a segurança das autotransfusões.

A indicação de uma autotransfusão está calcada em quatro itens fundamentais, a saber: Segurança: A autotransfusão é segura e isenta dos riscos e das complicações das transfusões homólogas, já apontados. Economia: o aspecto econômico é outro fundamento de nossa insistência na prática de autotransfusões. O seu custo quando se empregam métodos elementares, é mínimo. É preciso lembrar que numa autotransfusão, a matéria-prima - o sangue - é gratuita. Nas transfusões homólogas, além do gasto do material de coleta e embalagem, deve ser considerada a pecúnia ao doador. Disponibilidade: A oferta de sangue homólogo de doadores qualificados é sempre pequena. Quando se trata de tipos mais raros, esse problema se avoluma ainda mais. Para duas autotransfusões, seja de reinfusão ou de pré-depóstito, a disponibilidade é clara e evidente. Além disso, existe o aspecto da qualidade do sangue, que nas autotransfusões, mantém intactas as propriedades de viabilidade de seus componentes, de compatibilidade e até de temperatura( na pré-coleta imediata), o que nem sempre acontece nas transfusões homólogas. Amparo Jurídico: O estágio atual atingido pelas diversas formas de autotransfusão - a excelência de seus resultados e a segurança que oferece - já extravasou para o meio leigo. O médico que não oferece essa opção de reposição sangüínea a seu paciente cirúrgico está sujeito a problemas jurídicos, caso venha a ser obrigado a fazer transfusão homóloga. A segurança e o aspecto econômico das autotransfusões já foram também bastante divulgados no meio empresarial, sobretudo das companhias de seguros. As empresas de seguro médico já estão alertando profissionais e clientes com relação à adoção compulsória de autotransfusão em casos de operações que levam a sangramentos maiores. Recente sentença judicial na Alemanha condenou toda a equipe cirúrgica e o hospital ao pagamento de vultuosa indenização a um paciente submetido à cirurgia ortopédica, sabidamente sangrante, que recebeu uma transfusão homóloga ${ }^{20}$. Embora nenhuma reação adversa tenha ocorrido, a sentença condenatória baseou-se no risco a que foi submetido o paciente e no fato de o mesmo não ter sido previamente informado da existência da opção de autotransfusão no caso de sangramento cirúrgico maior.

Concluídas as considerações relativas às transfusões, não tememos afirmar que: da forma que evolui, a autotransfusão constituirá, em futuro bem próximo, um método terapêutico de uso compulsório para reposições sangüíneas, em vez de uma simples opção a escolher; as transfusões homólogas ficarão restritas a casos de extrema urgência em que o sangue do paciente tenha se perdido, sem chances de recuperação. As transfusões homólogas programadas desaparecerão do armamentário médico, ficando limitadas ao tratamento de hemopatias anemiantes clássicas, enquanto as pesquisas medicamentosas e de engenharia genética não as solucionarem. Por sua simplicidade, facilidade de manejo, multiaplicabilidade e aspecto econômico, a autotransfusão de pré-coleta imediata é atualmente, a nosso ver, a modalidade mais importante de transfusão homóloga. Nós a empregamos em praticamente todas as operações de grande e de médio porte.

\begin{abstract}
The practice of auto-transfusion is highly considered due to its safety and economic beneficts. It comprises two basic modalities: "Reinfusion" and "Pre-Collection" of blood. The reinfusion uses the patient's own blood spurted either from the patient's previous hemorrhage or during the surgery. The auto-transfusion of Pre-Collect uses the patient's blood tajen before surgery and it consists two basic modalities: The multiple Pre-Collection in which the blood is taken from the patient much before the scheduled sate of the surgery and the Pre-Collection done 10-30 minutes before surgery, which we presently designated as "Immediate Pre-Collection" (or PreDeposit for Immediate Utilization) which constitutes the basic topic of this essay (Rev. Col. Bras. Cir. 2008; 35(4): 259-263).
\end{abstract}

Key words: Blood Transfusion, Autologous; Blood Transfusion, Autologous /methods.

\section{REFERÊNCIAS}

1. Bogossian L. Manual de autotransfusão. Rio de Janeiro: Medsi; 1995.

2. Bogossian L. Aperfeiçoamento da técnica de reinfusão. Rev Bras Cir. 1960; 39:133.

3. Bogossian L. Papel atual da reinfusão sangüínea. Folha Med. 1986;92(4):223-8

4. Bogossian L, Gonçalves MDC, Bogossian AT. Autotransfusão. J Bras Med. 1988; 54(1/2):56.
5. Bogossian L, Bogossian AT, Bogossian TJT. Autotransfusão imediata: reinfusão. An Acad Nac Med. 1994;154(1):35-9.

6. Bogossian L. Traumatismos torácicos. Rio de Janeiro: Atheneu. 1976.

7. Bogossian L. Manual prático de pré e pós-operatório. $3^{\text {a }}$ ed. Rio de Janeiro: Rubio; 2007.

8. Nagino M, Kamiva J, Arai T, Nishio H, Ebata T, Nimura Y. One hundred consecutive hepatobiliary resections for biliary hilar malignancy: preoperative blood donation, blood loss, transfusion, and outcome. Surgery. 2005;137(2):148-55. 
9. Okabe T, Kim C, Yamanashi Y, Sakamoto A. [Anesthesia management for laparoscopic prostatectomy and open prostatectomy]. Masui. 2007;56(12):1404-7.

10. Gilcher RO, Orr MD. Intraoperative autotransfusion. Transfusion. 1975;15(5):520.

11. Deleuze P, Intrator L, Liou A, Contremoulins I, Cachera JP, Loisance DY. Complement activation and use of a cell saver in cardiopulmonary bypass. Asaio Trans. 1990;36(3):M179-81.

12. Schleinzer W. Autotransfusão. Conferência proferida no Hospital Universitário Clementino Fraga Filho em 17 de dezembro de 1992.

13. Bogossian AT, Ribeiro J, Bogossian L. Autotransfusão: importante complemento terapêutico das esplenopatias cirúrgicas. Rev Col Bras Cir. 1995;22(6):346-9.

14. Bogossian L, Bogossian TJT, Bogossian AT. Nova modalidade de autotransfusão. J Bras Med. 1989;57(5/6):73-4.

15. Bogossian AT, Ribeiro Filho J, Bogossian L. Reavaliação da autotransfusão de órgãos extirpados. Folha Med. 1995;110(1)558.

16. Chan AC, Blumgart LH, Wuest DL, Melendez JA, Fong Y. Use of preoperative autologous blood donation in liver resections for colorectal metastases. Am J Surg. 1998;175(6):461-5.

17. Dupuis JY, Bart B, Bryson G, Roblee J. Transfusion practices among patients who did and did not predonate autologous blood before elective cardiac surgery. CMAJ. 1999;160(7):987-1002.

18. Elawad AA, Jonsson S, Laurell M, Fredin H. Predonation autologous blood in hip arthroplasty. Acta Orthop Scand. 1991;62(3):218-22.

19. Johnson LB, Plotkin JS, Kuo PC. Reduced transfusion requirements during major hepatic resection with use of intraoperative isovolemic hemodilution. Am J Surg. 1998;176(6):608-11.

20. Lorentz A, Osswald PM, Schilling M, Jani L. [A comparison of autologous transfusion procedures in hip surgery]. Anaesthesist. 1991;40(4):205-13.

21. McVay PA, Fung HC, toy PT. Return of autologous blood donors. Transfusion. 1991;31(2):119-21.

22. Savoia HF, Metz J, Maxwell EL, Hauesler MN, Mellor J, Kiely S. Utilization of preoperative autologous blood donation in elective surgery. Am J Surg. 2002; 72(8):557-60.

23. Wang C, Lau W, Herst R, Drutz H, Fernandes B. Preoperative autologous blood deposition in support of gynaecological repair procedures. Transfus Med. 1998;8(1):23-7.

24. Sankarankutty AK, Teixeira AC, Souza FF, Mente ED, Oliveira GR, Almeida RCC, et al. Impact of blood salvage during liver transplantation on reduction in transfusion requirements. Acta Cir Bras. [serial on the Internet] 2006;21 Suppl 1. Available from URL: http://www.scielo.br/acb

25. Society of Thoracic Surgeons Blood Conservation Guideline Task Force, Ferraris VA, Ferraris SP, Saha SP, Hessel EA 2nd, Haan CK, Royston BD, Bridges CR, Higgins RS, Despotis G, Brown JR; Society of Cardiovascular Anesthesiologists Special Task Force on Blood Transfusion, Spiess BD, Shore-Lesserson L,
Stafford-Smith M, Mazer CD, Bennett-Guerrero E, Hill SE, Body S. Perioperative blood transfusion and blood conservation in cardiac surgery: the Society of Thoracic Surgeons and The Society of Cardiovascular anesthesiologists clinical practice guideline. Ann Thorac Surg. 2007;83(5 Suppl):S27-86.

26. Selo-Ojeme DO, Feyi-Waboso PA. Salvage autotransfusion versus homologous blood transfusion for ruptured ectopic pregnancy. Int J Gynecol Obstet. 2007;96(2):108-11. Epub 2007 Jan 19.

27. Bui LL, Smith AJ, Bercovici M, Szalai JP, Hanna SS. Minimising blood loss and transfusion requirements in hepatic resection. HPB. 2002;4(1):5-10.

28. Junqueira PC. Autotransfusão. Rio de Janeiro: BCG Computação Gráfica. 1992.

29. Matot I, Scheinin O, Jurim O, Eid A. Effectiveness of acute normovolemic hemodilution to minimize allogeneic blood transfusion in major liver resections. Anesthesiology. 2002;97(4):794-800.

30. Chen G, Zhang FJ, Gong M, Yan M. Effect of perioperative autologous versus allogeneic blood transfusion on the immune system in gastric cancer patients. J Zhejiang Univ Sci B. 2007;8(8):560-5.

31. Droste S, Sorensen T, Price T, Sayers M, Benedetti T, Easterling $\mathrm{T}$, Hendricks S. Maternal and fetal hemodynamic effects of autologous blood donation during pregnancy. Am J Obstet Gynecol. 1992;167(1):89-93.

32. Highmore W. Practical remarks on an overlooked source of blood supply for transfusion in post-partum haemorrhage. Lancet. 1874;1:89-92.

33. Simpson MB, Georgopoulos G, Orsini E, Eilert RE. Autologous transfusions for orthopaedic procedures at a children's hospital. J Bone Joint Surg Am. 1992; 74(5):652-8.

34. Walpoth BH, Volken U, Aeschbaker B et al. [Benefits and drawbacks of autologous blood predonation in patient undergoing cardiac surgery]. Schweiz Med Wochenschr. 1991;121(1):365.

35. Ascari WQ, Jolly PC, Thomas PA. Autologous blood transfusion in pulmonary surgery. Transfusion. 1968;8(2):111-5.

36. Singbartl G, Malgorzata S, Quoss A. Preoperative autologous blood donation - Part II. Adapting the predeposit concept to the physiological basics of erythropoiesis improves its efficacy. Minerva Anestesiol. 2007;73(3):153-60. Epub 2007 Jan 25.

Como citar este artigo:

Bogossian L, Bogossian AT. Autotransfusão de pré-coleta imediata. Rev Col Bras Cir. [periódico na Internet] 2008; 35(4). Disponível em URL: http://www.scielo.br/rcbc

Endereço para correspondência:

Rua Itacuruçá, 119/901

Tijuca - Rio de Janeiro/RJ

CEP: 20510-190

Telefax: (21) 2268-0653 